\title{
A Novel Retrieval-Dependent Memory Process Revealed by the Arrest of ERK1/2 Activation in the Basolateral Amygdala
}

\author{
-Emiliano Merlo, ${ }^{1,2}$ @Amy L. Milton, ${ }^{2}$ and $\odot$ Barry J. Everitt ${ }^{2}$ \\ ${ }^{1}$ Behavioural and Clinical Neuroscience Institute and ${ }^{2}$ Department of Psychology, University of Cambridge, Cambridge CB2 3EB, United Kingdom
}

Fully consolidated fear memories can be maintained or inhibited by retrieval-dependent mechanisms depending on the degree of re-exposure to fear cues. Short exposures promote memory maintenance through reconsolidation, and long exposures promote inhibition through extinction. Little is known about the neural mechanisms by which increasing cue exposure overrides reconsolidation and instead triggers extinction. Using auditory fear conditioning in male rats, we analyzed the role of a molecular mechanism common to reconsolidation and extinction of fear, ERK1/2 activation within the basolateral amygdala (BLA), after intermediate conditioned stimulus (CS) exposure events. We show that an intermediate re-exposure (four CS presentations) failed to activate ERK1/2 in the BLA, suggesting the absence of reconsolidation or extinction mechanisms. Supporting this hypothesis, pharmacologically inhibiting the BLA ERK1/2-dependent signaling pathway in conjunction with four CS presentations had no effect on fear expression, and the NMDA receptor partial agonist D-cycloserine, which enhanced extinction and ERK1/2 activation in partial extinction protocols (seven CSs), had no behavioral or molecular effect when given in association with four CS presentations. These molecular and behavioral data reveal a novel retrieval-dependent memory phase occurring along the transition between conditioned fear maintenance and inhibition. CS-dependent molecular events in the BLA may arrest reconsolidation intracellular signaling mechanism in an extinction-independent manner. These findings are critical for understanding the molecular underpinnings of fear memory persistence after retrieval both in health and disease.

Key words: ERK1/2; extinction; limbo; NMDAR; reconsolidation

Significance Statement

Consolidated fear memories can be altered by retrieval-dependent mechanisms. Whereas a brief conditioned stimulus (CS) exposure promotes fear memory maintenance through reconsolidation, a prolonged exposure engages extinction and fear inhibition. The nature of this transition and whether an intermediate degree of CS exposure engages reconsolidation or extinction is unknown. We show that an intermediate cue exposure session (four CSs) produces the arrest of ERK1/2 activation in the basolateral amygdala, a common mechanism for reconsolidation and extinction. Amnestic or hypermnestic treatments given in association with four CSs had no behavioral or molecular effects, respectively. This evidence reveals a novel retrieval-dependent memory phase. Intermediate degrees of CS exposure fail to trigger reconsolidation or extinction, leaving the original memory in an insensitive state.

\section{Introduction}

Persistent maladaptive associative memories are an essential aspect of chronic, recurrent anxiety disorders, including specific

\footnotetext{
Received Nov. 17, 2017; revised Jan. 11, 2018; accepted Jan. 16, 2018

Author contributions: EM designed research; E.M. and A.L.M. performed research; E.M. analyzed data; E.M., A.L.M., and B.J.E. wrote the paper.

This work was supported by the UK Medical Research Council (MRC) Programme Grant 9536855 to B.J.E. and A.L.M., and was conducted in the Department of Psychology and the MRC/Wellcome Trust Behavioural and Clinical Neuroscience Institute. E.M. is a Newton Alumnus of the Royal Society. We thank J. Berni, M. Pagani, E. Cahill, and J. Medina for critical reading of the manuscript.

The authors declare no competing financial interests.

Correspondence should be addressed to Dr. Emiliano Merlo, IFIBIO-Houssay, Universidad de Buenos AiresCONICET, Paraguay 2155, 1121 Buenos Aires, Argentina. E-mail: emimerlo@gmail.com.

E. Merlo's present address: IFIBI0-Houssay, Facultad de Medicina, Universidad de Buenos Aires—CONICET, 1121 Buenos Aires, Argentina.
}

phobias and post-traumatic stress disorder (PTSD; Parsons and Ressler, 2013). In human and nonhuman animals, retrieval of an associative memory by means of exposure to the fear conditioned stimulus (CS) can trigger either the maintenance or inhibition of stored memories, depending on the number or extent of cue presentations. A brief CS presentation, in the presence of a mismatch between what is expected and what actually occurs, leads to memory reconsolidation and the maintenance of the conditioned response (Pedreira et al., 2004; Kindt et al., 2009). By contrast, a large number of CS presentations at re-exposure triggers extinction and inhibition of the conditioned response 
(Pavlov, 1927; Bouton, 2004; Hermans et al., 2006). Intriguingly, although reconsolidation and extinction have opposite behavioral effects, both can be triggered by a similar event; a CS reminder without the unconditioned stimulus (US). Under extreme reminder conditions (brief or prolonged), memory trace dominance is evident since either reconsolidation or extinction is exclusively engaged (Eisenberg et al., 2003; Pedreira and Maldonado, 2003). Whether an intermediate degree of CS exposure triggers reconsolidation and/or extinction in a coexistent or mutually exclusive manner is unclear.

Understanding how fear memory reconsolidation and extinction relate to each other when retrieval is triggered by intermediate amounts of CS exposure is essential fully to understand the alternative effects of retrieval on memory persistence. Moreover, since CS exposure is an essential aspect of existing treatments for anxiety disorders (Vervliet et al., 2013), with growing interest in using pharmacological agents to potentiate their effectiveness (de Kleine et al., 2013), defining the boundary conditions - the characteristics of the transition from reconsolidation to extinction and the underlying molecular mechanisms-is essential for developing more effective therapies.

Even though reconsolidation and extinction of cued fear memory are dependent upon distinct networks within the brain, the basolateral amygdala (BLA) is a locus for both processes (Nader et al., 2000; Maren, 2015), and within the BLA these memory processes rely on both exclusive and common neural mechanisms. Thus, reconsolidation specifically requires synthesis of the immediate early gene Zif268 (Lee et al., 2005), while extinction specifically requires the synthesis of the protein phosphatase calcineurin (Merlo et al., 2014). However, both processes rely on the antecedent activation of NMDA receptors (NMDARs; Lee and Kim, 1998; Milton et al., 2013) and the extracellular signal-regulated kinase 1/2 (ERK1/2) signaling pathway (Duvarci et al., 2005; Herry et al., 2006). Thus, while reconsolidation and extinction are initiated by similar extracellular and intracellular events, the transcriptional and translational events underlying each memory process are unique (Mamiya et al., 2009; Merlo et al., 2014).

Because BLA ERK1/2 is activated by both fear memory reconsolidation and extinction, measuring CS exposure-dependent BLA phosphorylated ERK1/2 (pERK1/2) levels provides an ideal molecular marker to distinguish between the mutually exclusive or gradual coexistence hypotheses of these opposing processes (Pérez-Cuesta and Maldonado, 2009). Thus, if the transition is gradual, with both memory processes being partially engaged, pERK1/2 should be increased by an intermediate number of CS presentations. By contrast, a failure to affect BLA pERK1/2 levels by intermediate retrieval conditions would support a mutually exclusive three-phase transition. Therefore, in the present experiments we measured BLA pERK1/2 levels and the behavioral effect of ERK1/2 signaling cascade inhibition when fear memory was retrieved by presenting an intermediate number of CSs. We hypothesized that in rats with a fully consolidated auditory fear memory an intermediate number of CSs would fail to activate ERK1/2 in the BLA, supporting the three-phase transition hypothesis.

We demonstrate here that four CS presentations had no effect on BLA pERK1/2 levels and left the fear memory insensitive to ERK1/2 blockade in the BLA. We thereby reveal a mutually exclusive relationship between reconsolidation and extinction and show that the transition between them as a result of increasing CS exposure is explained by a three-phase model.

\section{Materials and Methods}

Animals. Two hundred and fifty adult male Lister Hooded rats weighing 250-300 g (Charles River; RRID:RGD_2312466) were used. All animals were kept under a $12 \mathrm{~h}$ light/dark cycle (lights off at 7:00 A.M.) and provided with food and water ad libitum except for during behavioral procedures. All animal procedures were conducted in accordance with the European Union (EU) legislation on the protection of animals used for scientific purposes (Directive 2010/63/EU) and the research was regulated under the Animals (Scientific Procedures) Act 1986 Amendment Regulations 2012 following ethical review by the University of Cambridge Animal Welfare and Ethical Review Body.

Surgeries. Rats were anesthetized with ketamine hydrochloride (100 $\mathrm{mg} / \mathrm{kg}$; Ketaset, Fort Dodge Animal Health) and xylazine $(9 \mathrm{mg} / \mathrm{kg}$; Rompun, Bayer), and implanted with 22-gauge stainless steel bilateral indwelling guide cannulae (Plastics One) aimed at the BLA. The coordinates were $2.6 \mathrm{~mm}$ posterior to bregma, $4.5 \mathrm{~mm}$ lateral to the midline, and $3.6 \mathrm{~mm}$ ventral to dura mater. Stainless steel obturators were inserted to maintain patency during recovery and in between infusions.

Intracranial microinfusions. Infusions were performed as described previously (Merlo et al., 2014), with injectors extending $4 \mathrm{~mm}$ beyond the guide cannulae. Before behavioral testing, animals were habituated to the infusion procedure by the administration of $0.1 \mu \mathrm{l}$ of sterile saline (SAL) solution per side $(0.25 \mu \mathrm{l} / \mathrm{min})$. U0126 (Sigma-Aldrich) was dissolved in 5\% DMSO and 6\% Tween 80 in $100 \mathrm{~mm}$ sterile PBS (PBS, pH 7.2) to a final concentration of $2 \mu \mathrm{g} / \mu \mathrm{l}$. A total of a $0.5 \mu \mathrm{l}$ of U0126 or vehicle (VEH; 5\% DMSO and 6\% Tween 80 in $100 \mathrm{~mm}$ sterile PBS) solution per side $(0.25 \mu \mathrm{l} / \mathrm{min})$ were infused $30 \mathrm{~min}$ before re-exposure session.

Behavioral procedures. Animals were initially individually habituated to the conditioning box (Paul Fray) for $2 \mathrm{~h}$. On the training day, rats were placed in the box and after 25 min received an auditory CS presentation ( $60 \mathrm{~s}$ clicker, $10 \mathrm{~Hz}, 80 \mathrm{~dB}$ ) that was coterminous with the presentation of a scrambled footshock (US: $0.5 \mathrm{~mA}, 0.5 \mathrm{~s}$ ) delivered through the grid floor. The training session consisted of two CS-US presentations with an intertrial interval (ITI) of $5 \mathrm{~min}$. Twenty-four hours later, the rats were returned to the box and presented with $1,4,7$, or 10 CS presentations (ITI, $1 \mathrm{~min}$ ). Twenty-four hours later, animals were again returned to the conditioning box and presented with one CS.

Drug injection. The NMDAR partial agonist D-cycloserine (DCS; Sigma-Aldrich) or the noncompetitive receptor antagonist MK-801 was dissolved in sterile saline for intraperitoneal injection $(1 \mathrm{ml} / \mathrm{kg})$. The doses of $15 \mathrm{mg} / \mathrm{kg}$ (DCS) or $0.1 \mathrm{mg} / \mathrm{kg}$ (MK-801) were selected on the basis of their mnemonic effects on prior experiments on the reconsolidation and extinction of fear memory (Merlo et al., 2014). Drug or saline solution injections were given $30 \mathrm{~min}$ before CS presentation sessions.

Protein extraction and Western blotting. Rats were killed by carbon dioxide inhalation followed by neck dislocation. The brains were rapidly removed and snap frozen on dry ice before storage at $-80^{\circ} \mathrm{C}$. Cytosolic protein preparation, quantification, and separation were performed as described previously (Merlo et al., 2014). Blots were probed with the following: mouse anti-ERK1/2 (1:5000; catalog \#610124, BD Biosciences; RRID:AB_397530), rabbit anti-phospho-p44/42 MAPK (ERK1/2; Thr 202/Tyr 204; D13.14.4E; 1:500; Cell Signaling Technology; RRID: AB_10694057), mouse anti- $\beta$-actin (1:50,000; catalog \#AC-15, Abcam; RRID:AB_2223210), goat anti-rabbit-HRP (1:10,000; Sigma-Aldrich) and rabbit anti-mouse-HRP (1:25,000; Sigma-Aldrich) diluted in Trisbuffered saline solution containing $0.1 \%$ Tween-20. A chemiluminescent signal was induced using an enhanced chemiluminescent reagent (GE Healthcare), and images were captured using a cooled CCD camera (ChemiDoc-It, UVP). Signal analysis and quantification were performed using ImageJ software (version 1.47a, National Institutes of Health; RRID:SCR_003070). Each primary antibody working concentration was adjusted to deliver a linear relationship between the amounts of loaded protein in the blot versus signal intensity.

Experimental design and statistical analysis. All training, CS presentation, and test sessions were video recorded for off-line behavioral analysis. The percentage of time freezing (i.e., the absence of movement except for breathing) during the $1 \mathrm{~min}$ before and during the $1 \mathrm{~min}$ CS was 
A

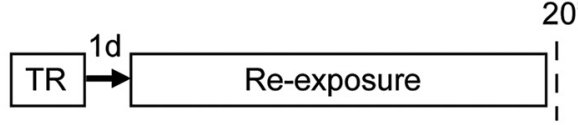

B

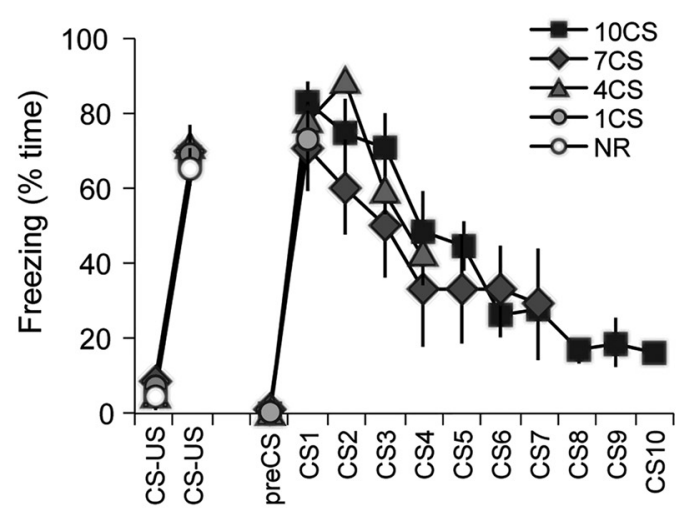

C
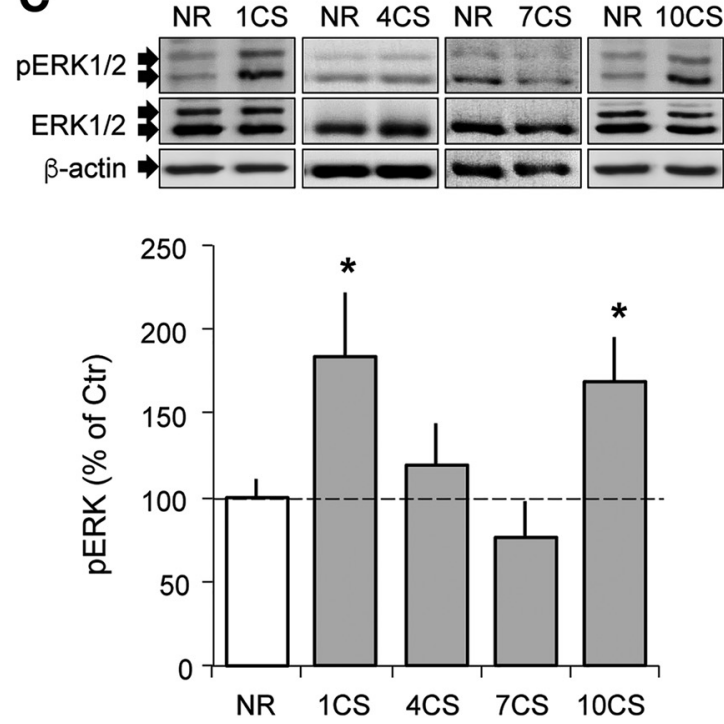

Figure 1. Intermediate cue exposure fails to activate BLA ERK1/2, a molecular marker of fear memory reconsolidation and extinction. $A$, Experimental design. Rats were fear conditioned with two CS-US pairings. Twenty-four hours after training, animals were divided into the following five groups: NR control, and 1, 4, 7, and 10 CS presentations (1CS, 4CS, 7CS, or 10CS, respectively). Twenty minutes after the first CS presentation, or straight from the home cage, animals were killed and BLA cytosolic protein extracts prepared. $\boldsymbol{B}$, Mean ( \pm SEM) percentage of time spent freezing during cued fear conditioning and at different number of cue exposure sessions (NR, $n=23 ; 1 C S, n=12 ; 4 C S, n=10 ; 7 C S, n=8 ; 10 C S, n=12$ ). C, Representative Western blot picture and analysis of pERK1/2 levels in the BLA for NR, 1CS, 4CS, 7CS, and 10CS groups. Mean relative optical density as the percentage of NR ( \pm SEM) shows that pERK1/2 is increased after 1 or $10 C S$ presentations, but is unchanged after 4 or 7 CSs. TR, Training session. ${ }^{*} p<0.05$.

manually scored from the videos at $5 \mathrm{~s}$ intervals by an observer blind to the treatment. Statistical analyses of behavioral quantifications were performed using one-way, two-way, or repeated-measures ANOVAs, with "Group" as the between-subjects factor and CS-US or CS as the withinsubjects factor. Tukey's post hoc test comparisons were used for further analysis. Any deviations from sphericity were corrected using the Greenhouse-Geisser correction if $\varepsilon<0.75$, and the Huynh-Feldt correction if $\varepsilon>0.75$ (Cardinal and Aitken, 2006). For Western blot data analysis, optical density (OD) values and the band areas were obtained for each microdissected basolateral amygdala cytosolic sample for both target protein (pERK1/2, ERK1/2) and the $\beta$-actin loading control. Each pERK1/2 OD value was normalized to its corresponding ERK $1 / 2$ and $\beta$-actin OD value. These normalized OD values for the experimental groups were normalized to the nonreactivated (NR) control group mean OD value, and then averaged for each condition. To minimize variability across membranes, each Western blot membrane included at least three different samples of each experimental and control groups. Each Western blot quantification was performed twice to ensure replicability. Molecular data were analyzed using one-way ANOVA with Dunnett's test for post hoc comparisons (Fig. 1C). For Western blot quantifications presented in Figure 4, OD values for SAL/drug-treated animals were normalized to the mean OD of their respective NR-SAL or NR-drug group. These data were analyzed using Student's $t$ test. In both cases, Group was the betweensubjects factor. Tests were performed using SPSS (IBM; RRID:SCR_002865) and JASP (University of Amsterdam, Amsterdam, The Netherlands; RRID:SCR_015823).

\section{Results}

\section{Intermediate CS exposures fail to active BLA ERK1/2}

Rats were fear conditioned by two pairings of an auditory clicker (CS) and a footshock (US). Animals were divided according to the treatment received during re-exposure day into five experimental groups as follows: NR control group; and exposure to 1 (1 CS), 4 (4 CSs), 7 (7 CSs), or 10 (10 CSs) unreinforced 1-minlong CS presentations (Fig. $1 A$ ). A mixed ANOVA indicated that all prospective groups showed similar acquisition of fear conditioning, with an effect of trial (CS-US ${ }_{1}$ vs CS-US ${ }_{2}: F_{(1,60)}=468.3$,

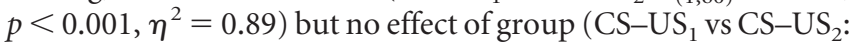

$\left.F_{(4,60)}=0.24, p=0.91\right)$ or interaction $\left(\mathrm{CS}-\mathrm{US}_{1}\right.$ vs CS-US $: F_{(4,60)}=$ $0.10, p=0.98$; Fig. $1 B$ ).

Twenty-four hours later, animals were either re-exposed to the different numbers of CSs or remained in their home cages as controls (Fig. 1B). Freezing in response to the first CS gives a measure of fear memory retention that can be assessed for all CS-exposed groups, to ensure that training was equivalent. All CS-exposed animals showed high levels of freezing to the first CS (pre-CS vs $\mathrm{CS}_{1}: F_{(1,38)}=378.2, p<0.001, \eta^{2}=0.91$ ) with no effect of Group (pre-CS vs $\mathrm{CS}_{1}: F_{(3,38)}=0.52, p=0.67$ ) or any interaction (pre-CS vs $\mathrm{CS}_{1}: F_{(3,60)}=0.72, p=0.55$ ), indicating similar levels of fear memory retention across groups. Repeatedmeasures ANOVAs showed significant effects of trials on freezing level in $4 \mathrm{CS}\left(F_{(1.52,13.71)}=20.18, p<0.001, \eta^{2}=0.69\right), 7 \mathrm{CS}$ $\left(F_{(1.60,11.23)}=5.31, p=0.03, \eta^{2}=0.43\right)$, and $10 \mathrm{CS}$ groups $\left(F_{(2.71,29.86)}=33.31, p<0.001, \eta^{2}=0.75\right)$.

Twenty minutes after the presentation of the first CS, or immediately following removal from the home cage (NR group), animals were killed and cytosolic protein extracts from the BLA prepared. A one-way ANOVA of BLA pERK1/2 levels quantified by Western blots showed that the groups differed in their pERK1/2 level $\left(F_{(4,60)}=3.55, p=0.01, \eta^{2}=0.19\right)$. Post hoc comparisons (Dunnett's test) showed that pERK1/2 was increased after 1 or $10 \mathrm{CS}$ presentations ( $p=0.01$ and $p=0.04$, respectively) but remained at basal levels after 4 or 7 CSs $(p=0.62, p=0.97$, respectively; Fig. $1 C)$.

We have previously shown that under these specific retrieval conditions, the presentation of one unreinforced CS leads to reconsolidation of the original memory, whereas 10 CS presentations leads to the formation of a new inhibitory extinction memory (Lee et al., 2006; Merlo et al., 2014). Thus, these results confirmed the engagement within the BLA of the ERK1/2 signaling pathway under extreme reminder conditions, with both the reconsolidation-inducing and extinction-inducing CS protocols resulting in kinase activation. By contrast, intermediate reminders of 4 or 7 CS presentations did not result in BLA ERK1/2 

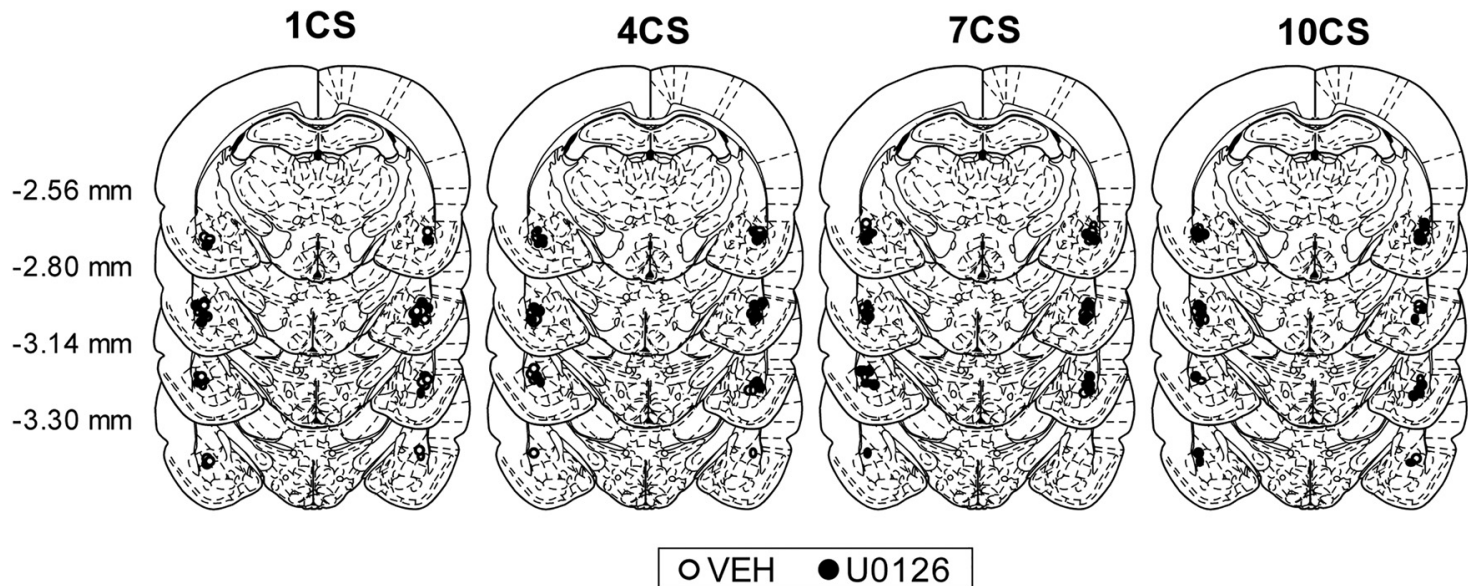

O VEH

Figure 2. Injector tip placements within the basolateral amygdala for each experimental condition: 1,4,7, and 10 CSs. Vehicle: open circles; U0126: closed circles. This figure was modified, with permission, from Paxinos and Watson (1998).

activation. These observations support our main hypothesis indicating that a common synaptic plasticity mechanism engaged during either reconsolidation or extinction of fear memory is not recruited under these intermediate CS exposure conditions.

\section{ERK1/2 signaling cascade blockade during intermediate CS exposure has no effect on memory}

The lack of ERK1/2 activation after four or seven CS presentations suggests that the CS exposure conditions leading to reconsolidation or extinction are separated by a degree of CS exposure during which no ERK-dependent synaptic plasticity mechanisms are engaged within the BLA. Alternatively, it is possible that during the transition period, both reconsolidation and extinction are taking place, but in a gradual or more subtle way that is undetectable in BLA pERK1/2 levels. To distinguish between these two alternatives, we analyzed the effect of specific inhibition of the ERK1/2 signaling pathway on fear memory after presentation of four or seven CSs. To control for the amnestic effect of the pharmacological manipulation, the experiment included groups of animals treated while undergoing either reconsolidation or extinction triggered by extreme CS exposure levels (1 or 10 CSs, respectively).

Rats with bilateral cannulae targeting the BLA (Fig. 2) were fear conditioned as before. Twenty-four hours later, animals received an intra-BLA infusion of vehicle or the ERK1/2 inhibitor U0126 30 min before the presentation of $1,4,7$, or 10 CSs. One day later, fear memory was assessed by the presentation of one CS (Fig. 3A). Intra-BLA infusion of U0126 had no acute behavioural effect during CS exposure sessions, with similar freezing levels across groups for the first CS (Group: F $(3,71)=1.55, p=0.21$; Drug: $\mathrm{F}(1,71)=0.03, p=0.87$; Group $\times$ Drug: $\mathrm{F}(3,71)=0.35$, $p=0.79$ ), or along the session (4CS groups Drug: $\mathrm{F}(1,19)=$ $0.21, p=0.65, \mathrm{CS} \times$ Drug: $\mathrm{F}(3,57)=1.13, p=0.35 ; 7 \mathrm{CS}$ groups Drug: $\mathrm{F}(1,18)=0.46, p=0.51, \mathrm{CS} \times$ Drug: $\mathrm{F}(3.28,59.07)=$ $0.94, p=0.43$; 10CS groups Drug: $\mathrm{F}(1,15)=0.87, p=0.37$, CS $\times$ Drug: $\mathrm{F}(2.81,42.19)=1.18, p=0.33$; Figure $3 B)$. At test, a two-way ANOVA revealed no overall effect of the number of $\operatorname{CSs}\left(F_{(3,71)}=1.87, p=0.14\right)$ or of ERK1/2 inhibition $\left(F_{(1,71)}=\right.$ $0.03, p=0.86$ ), but a significant CS-Drug interaction, indicating differential effects of ERK1/2 inhibition with different levels of CS presentation (CS $\times$ Drug: $F_{(3,71)}=4.06, p=0.01, \eta^{2}=0.15$; Fig. $3 C)$. Simple main effects analysis showed an effect of drug for 1 $\operatorname{CS}\left(F_{(1,19)}=9.93, p=0.005, \eta^{2}=0.34\right)$ and 10 CS conditions
$\left(F_{(1,17)}=5.10, p=0.04, \eta^{2}=0.25\right)$, but not for $4 \mathrm{CS}$ groups $\left(F_{(1,19)}=0.004, p=0.95\right)$ or $7 \mathrm{CS}$ groups $\left(F_{(1,18)}=0.32, p=\right.$ $0.58)$.

Given that some of our experimental conditions tested the null hypothesis $\left[H_{0}\right.$; i.e., the absence of U0126 effect (four and seven CSs)], we also analyzed these data using Bayesian statistics, a method not biased against $H_{0}$ (Wagenmakers, 2007; Rouder et al., 2009). This revealed that the alternative hypothesis $\left(H_{1}\right)$ was 8.37 times more likely in animals receiving 1 CS, and 2.04 times more likely in animals receiving 10 CSs (1 CS data: (Bayesian Factor) $\mathrm{BF}_{01}=0.12 ; \mathrm{BF}_{10}=8.37 ; 10 \mathrm{CS}$ data: $\mathrm{BF}_{01}=0.49 ; \mathrm{BF}_{10}=$ 2.04). In contrast, in animals receiving four $\mathrm{CSs} H_{0}$ was 2.55 times more likely, whereas in animals receiving seven CSs the null hypothesis was 2.25 times more likely (four CS data: $\mathrm{BF}_{01}=2.55$; $\mathrm{BF}_{10}=0.39$; seven CS data: $\left.\mathrm{BF}_{01}=2.25 ; \mathrm{BF}_{10}=0.45\right)$. This analysis is consistent with the frequentist analysis presented above, further confirming the absence of an amnestic effect of U0126 when administered before four or seven CS presentations.

In addition to confirming previous observations on the requirement for BLA ERK1/2 in auditory fear memory reconsolidation and extinction (Duvarci et al., 2005; Herry et al., 2006) in the 1 CS and 10 CS groups, respectively, these data indicate that the same intervention had no behavioral effect when animals are exposed to an intermediate number of CSs.

The molecular and behavioral data presented so far indicate that both the four and seven CS exposure conditions not only fail to trigger fear memory labilization but also are insufficient to engage extinction. To further test the memory process dominance or absence in these two transitional stages, we conducted the next series of experiments evaluating the effect of NMDAR activity modulation on the effective or ineffective activation of pERK1/2 in the BLA.

BLA pERK1/2 enhancement by DCS during intermediate CS presentations distinguishes between sensitive and insensitive transitional states

The experiments presented here had the following two objectives: (1) to study the NMDAR activity dependence of BLA ERK1/2 activation during reconsolidation or extinction of fear memory; and (2) to evaluate the effect of NMDAR activity enhancement on the lack of BLA ERK1/2 activation seen after four or seven CS presentations. Systemic NMDAR activity manipulations were 
A

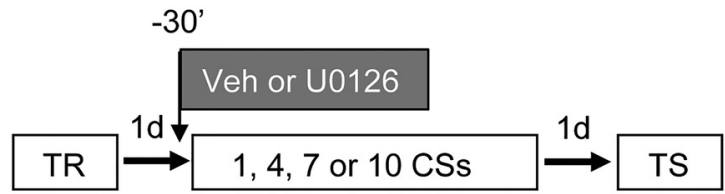

B
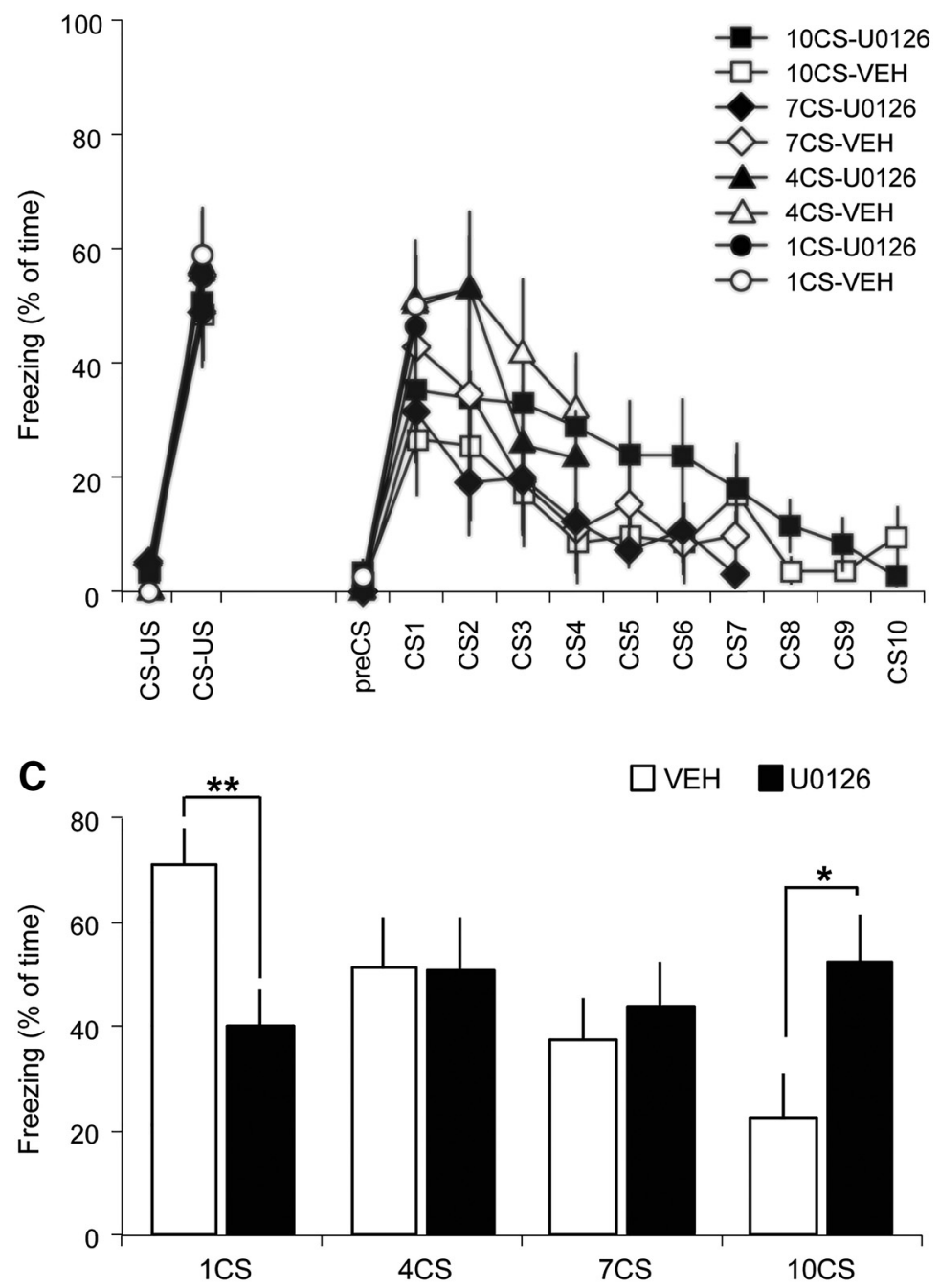

Figure 3. Intra-BLA administration of U0126, a specific inhibitor of ERK1/2 pathway, has no behavioral consequences during intermediate CS exposure. $A$, Experimental design. Animals were trained with two CS-US pairings. Twenty-four hours later, they were injected with vehicle or U0126 (1 $\mu \mathrm{g} / \mathrm{side})$ and within each drug condition divided into four groups (1CS, 4CS, 7CS, and 10CS) depending on the number of cue presentations. Twenty-four hours later, all the animals were tested for fear memory with the presentation of one CS (1CS VEH and 4CS U0126, $n=11 ; 10 C S$ VEH, $n=7$; remaining groups; $n=10$ per group). $B, C$, Mean percentage of time spent freezing ( \pm SEM) at $(S$ exposure sessions $(\boldsymbol{B})$ or long-term memory test $(\boldsymbol{C})$ are shown. TS, Test session. ${ }^{*} p<0.05 ;{ }^{* *} p<0.01$.

performed using the NMDA receptor antagonist MK-801 or partial agonist DCS.

To test the requirement for NMDAR activity in reconsolidationor extinction-induced BLA ERK1/2 activation, rats were fear conditioned as before. Twenty-four hours later, animals were injected systemically with saline or MK-80130 min before 1 or 10 CS presentations. Twenty minutes after the first CS presentation, animals were killed and their brains removed. NR control groups received the same intraperitoneal injections, but were returned to their home cages and killed 50 min later. To test for the effect of CS exposure on BLA pERK1/2 level depending on drug condition, we performed unpaired $t$ tests on Western blot data. This analysis shows that one CS presentation induced an increase in BLA pERK $1 / 2$ level in the saline group $\left(t_{(14)}=3.08 ; p=\right.$ $0.004)$, whereas there was no change in the presence of MK-801 $\left(t_{(14)}=0.07 ; p=\right.$ 0.47; Fig. 4A). Similarly, there was a pERK $1 / 2$ increase in rats injected with saline and exposed to $10 \mathrm{CSs}\left(t_{(14)}=2.83\right.$; $p=0.006)$, but not in rats treated with MK-801 $\left(t_{(14)}=1.04 ; p=0.16\right.$; Fig. $\left.4 B\right)$. These data are consistent with the disruption of reconsolidation or extinction by NMDAR blockade under extreme CS presentation conditions, and demonstrate that NMDAR activation is required for the increase in pERK1/2 associated with both of these mnemonic processes.

In parallel, we analyzed the effect of NMDAR activity enhancement on the BLA ERK1/2 activation state after four or seven CS presentations, hypothesizing that if there is a mnemonic-insensitive period engaged by these levels of CS presentation, then it should not be possible to modulate pERK1/2 level by enhancing NMDAR activity. Twenty-four hours after fear conditioning, rats were injected with saline or DCS $30 \mathrm{~min}$ before four or seven CS presentations. Twenty minutes after the first CS presentation, animals were killed and their brains removed. Nonreactivated control groups were treated as before. Animals exposed to four CS presentations showed no change in BLA pERK1/2 levels in both the saline $\left(t_{(8)}=\right.$ $1.09 ; p=0.16)$ or DCS condition $\left(t_{(8)}=\right.$ 0.78 ; $p=0.23$; Fig. $4 C$ ). By contrast, animals exposed to seven CS presentations showed no difference in pERK1/2 levels when injected with saline $\left(t_{(8)}=0.29\right.$; $p=0.39$ ), but showed a significant increase in the activated kinase levels when injected with DCS $\left(t_{(8)}=2.12\right.$; $p=0.03$; Fig. 4D).

These results indicate that ERK1/2 activation by reconsolidation or extinction of fear memory is dependent on NMDAR activity. Also, they show that enhancement of NMDAR activity increased pERK1/2 levels only in the seven CS group, suggesting that the two transitional states induced by four or seven CSs engage qualitatively different neural mechanisms.

\section{Discussion}

We have previously proposed that the transition from reconsolidation to extinction induced by increasing CS exposure conformed to a three-phase transition model that is characterized by an intermediate insensitive memory phase (Merlo et al., 2014). Since that hypothesis was based solely on behavioral observations following systemic pharmacological manipulations, it was possible that the lack of a memory modulation effect after 
A
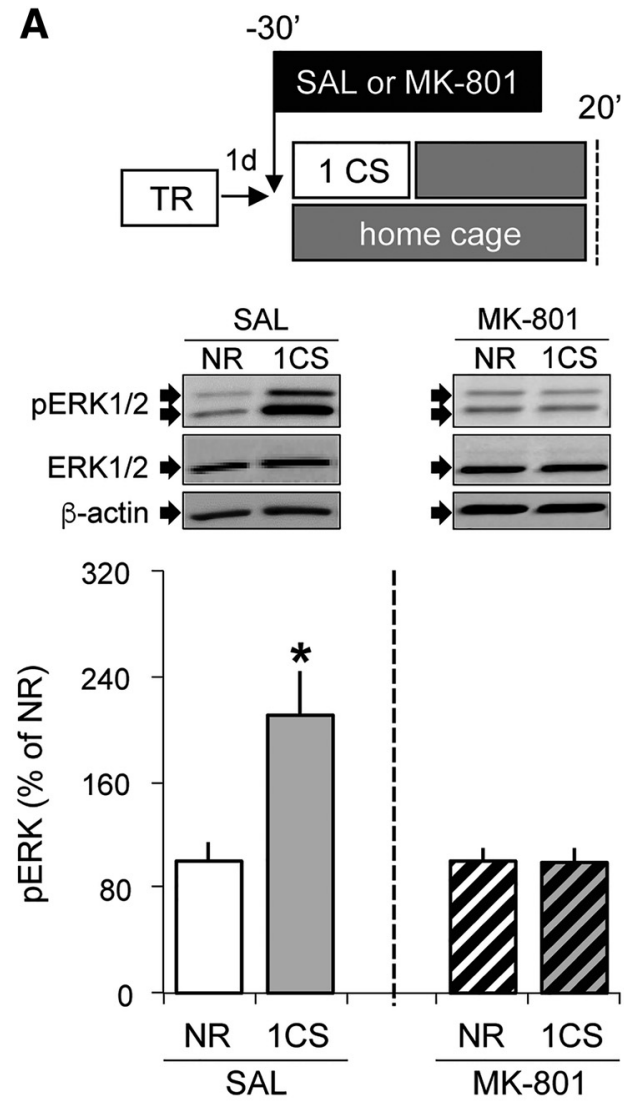

C
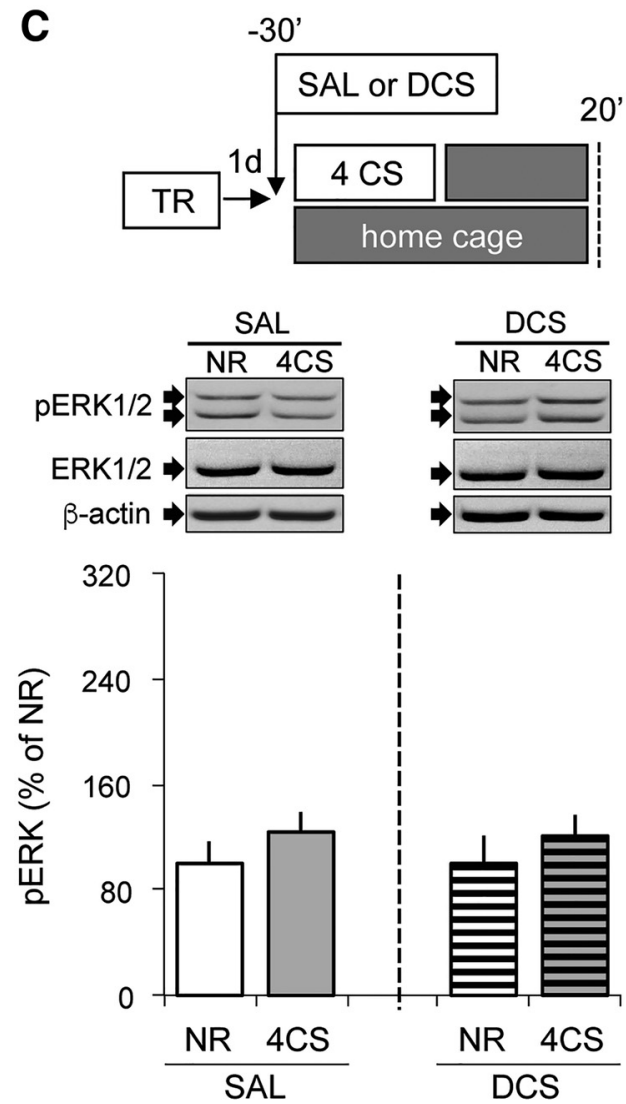

B
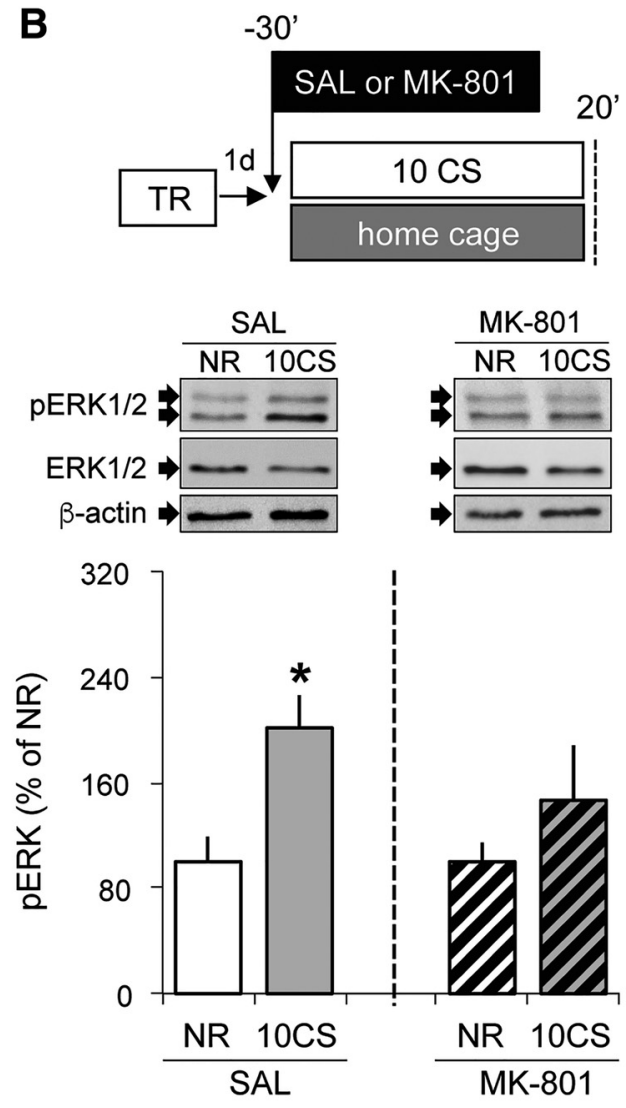

D
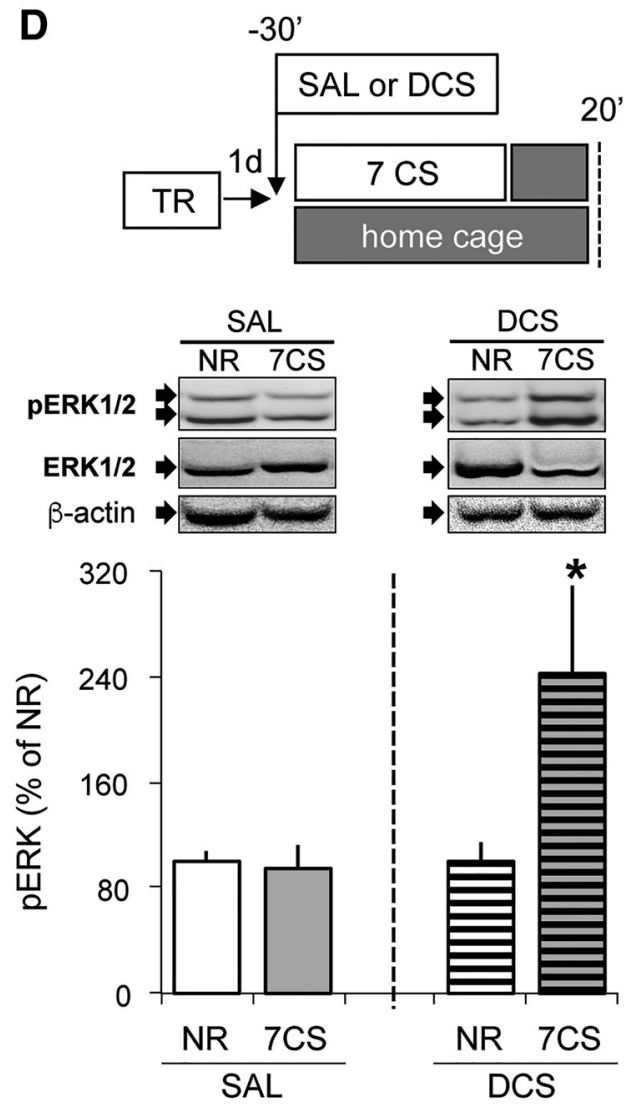

Figure 4. NMDAR-dependent BLA ERK1/2 activation distinguishes between sensitive and insensitive transitional states between fear memory reconsolidation and extinction. $\boldsymbol{A}$, Experimental design. Twenty-four hours after training, animals were intraperitoneally injected with saline or MK-801 (0.1 mg/kg) and then exposed to one CS (1CS groups) or returned to the home cage (NR groups). Fifty minutes after the injection, the animals were killed and BLA cytosolic protein extracts prepared. Representative Western blot pictures. Bar graph (Figure legend continues.) 
four CSs was the result of simultaneous and opposing effects on both reconsolidation and extinction mechanisms that were partially engaged in the brain. In the present study, we used a complementary molecular biological and behavioral approach along with more specific intra-BLA pharmacological manipulations to investigate the existence of this novel retrieval-dependent memory process triggered by intermediate degrees of cue exposure at memory retrieval. We show that activation of the BLA ERK1/2 signaling pathway, a well established molecular marker for reconsolidation and extinction, reveals a mutually exclusive, threephase transition between these memory processes when nonreinforced CS exposure is increased. Whereas extreme reminder conditions that trigger either fear memory reconsolidation or extinction ( 1 or $10 \mathrm{CSs}$ ) resulted in an increase in BLA pERK1/2, intermediate reminders ( 4 or 7 CSs) failed to alter BLA pERK1/2 levels. This transition mode was corroborated by pharmacological manipulation of the ERK1/2 signaling pathway or NMDAtype glutamate receptor activity. Thus, intra-BLA inhibition of the ERK1/2-dependent signaling cascade by U0126 disrupted both reconsolidation and extinction, but had no behavioral effect under intermediate CS exposure protocols. However, systemic DCS treatment revealed a distinction between four and seven CS conditions, since it failed to affect BLA ERK1/2 activation at four CS, but increased it at seven CSs, when it is also able to enhance extinction (Merlo et al., 2014).

It is well established that the ERK1/2 signaling pathway within the BLA is an essential mechanism underlying consolidation, reconsolidation and extinction of auditory fear memory (Schafe et al., 2000; Duvarci et al., 2005; Herry et al., 2006). In particular, it has been shown that intra-BLA administration of U0126 does not permanently damage the BLA, and has an amnestic effect only if the fully consolidated fear memory is retrieved (Duvarci et al., 2005). Here we show that during an intermediate reminder this amnestic manipulation is without effect even in presence of fear memory retrieval (Fig. 3). Although retrieval is a necessary condition for ERK1/2 blockade in the BLA to have an amnestic effect, it is not sufficient. U0126 can exert its amnestic effect only when infused into the BLA when either reconsolidation or extinction has been engaged.

The effect of four CS presentations on the fear response toward the CS at test is not significantly different from that of the one CS group, but is invariably at a lower level (Fig. 3C, 1CS-VEH vs 4CS-VEH; Merlo et al., 2014). A similar extinction-independent decrease in a contextual fear response was observed at test after intermediate context exposure (Cassini et al., 2017). It remains to be determined how four CSs affect the original fear memory. They may act to reduce the stored CS-US contingency information through a mechanism independent of extinction, affecting

\section{$\leftarrow$}

(Figure legend continued.) shows the mean BLA level of pERK1/2 ( \pm SEM) as a percentage of NR group ( $n=8$ per group). Open bars, saline injection; striped bars, MK-801 injection. $\boldsymbol{B}$, Experimental design and bar graph: same as for $\boldsymbol{A}$, but after intraperitoneal injection the animals were either returned to the home cage (NR groups) or exposed to $10 \mathrm{CS}$ presentations (10CS groups, $n=8$ per group). $C$, Experimental design. Twenty-four hours after training, animals were intraperitoneally injected with saline or DCS $(15 \mathrm{mg} / \mathrm{kg})$ and then exposed to four CSs (4CS groups) or returned to the home cage (NR groups). Fifty minutes after the injection, the animals were killed and BLA cytosolic protein extracts prepared. Representative Western blot pictures. Bar graph shows the mean BLA level of pERK1/2 ( \pm SEM) as a percentage of NR group ( $n=5$ per group). Open bars, saline injection; striped bars, DCS injection. $D$, Experimental design and bar graph: same as for $\boldsymbol{C}$, but after intraperitoneal injection the animals were either returned to the home cage (NR groups) or exposed to seven CS presentations (7CS groups, $n=5$ per group). ${ }^{*} p<0.05$ vs NR group receiving the same intraperitoneal injection. the original memory trace in the absence of reconsolidation. Such a mechanism could produce conditioned fear reduction without reinstatement, renewal, or spontaneous recovery. Interestingly, seven CS presentations did produce a reduction of fear at test compared with the one CS group, suggestive of the early engagement of extinction mechanisms. Even though this is an indirect observation, it supports the qualitative difference between these two intermediate reminder conditions that were discussed above.

As shown here, the molecular biological or behavioral analyses conducted separately do not fully reveal the complexity of memory mechanisms taking place during the transition from reconsolidation to extinction. This exemplifies the necessity of combining these approaches in order fully to understand these mechanisms. Failure to promote ERK1/2 activation in the BLA after an intermediate reminder does not provide a true biomarker of memory in limbo since this was also observed in both four and seven CS conditions. The lack of a memory-enhancing effect of BLA ERK1/2-positive modulation following DCS administration is therefore further evidence of the limbo state. These two sources of experimental evidence, while affirming the existence of the limbo state, cannot rule out the possibility of a third mnemonic process interposed between reconsolidation and extinction, and this warrants further detailed experimental investigation.

\section{Reconsolidation and extinction: mutually exclusive versus coexistence hypotheses}

In an attempt to distinguish between these alternative hypotheses, previous reports have investigated the pharmacological effects of amnestic treatments during intermediate CS exposure. In medaka fish, exposure to the amnestic agent 3-aminobenzoic acid ethyl ester (that can prevent both reconsolidation and extinction) during an intermediate CS exposure session had no behavioral effects (Eisenberg et al., 2003). In rats, systemic administration of an NMDAR agonist or antagonist had no effect when given in association with an intermediate fear or appetitive memory retrieval session (Flavell and Lee, 2013; Merlo et al., 2014). Finally, a period of insensitivity has been reported for a fear memory in humans after an intermediate exposure session and oral administration of the $\beta$-adrenoceptor antagonist propranolol, which prevents reconsolidation after brief memory reactivation (Sevenster et al., 2014). Even though these are consistent observations from fish to humans, the lack of specificity of the pharmacological manipulations used in these studies makes it difficult to distinguish between the two alternative memory dominance hypotheses (Pérez-Cuesta and Maldonado, 2009).

Here we present molecular and behavioral evidence indicating that reconsolidation and extinction do not coexist and that there is a limbo memory state when BLA ERK1/2 and fear expression are immune to amnestic and hypermnestic manipulations. The combination of precise parametric control of CS exposure levels with a well characterized amnestic treatment, the ERK1/2 inhibitor U0126 (which blocks both reconsolidation and extinction under extreme CS exposure conditions) allows us to reject the coexistence hypothesis and reveal the existence of an impervious, limbo memory state.

The parametric conditions that determine the engagement of alternative retrieval-dependent memory processes also depend on memory acquisition conditions (i.e., US intensity, CS duration) or memory age. Stronger or older fear memories require an extended CS re-exposure event to engage memory reconsolidation when compared with a younger or weaker memory (Suzuki et al., 2004). Contextual fear memories trained with longer context exposure bouts before shock presentation showed a 
rightward shift in the three-phase transition profile of reconsolidation, "limbo," and extinction (Alfei et al., 2015). Moreover, under the experimental conditions used here, it was the intermediate number of four CS presentations that failed to trigger reconsolidation or extinction. It is therefore possible that the degree of CS exposure necessary for limbo engagement will be sensitive to conditions such as US intensity at training, CS frequency at re-exposure, or total CS exposure time.

\section{Molecular markers of memory in limbo}

Activation of the ERK1/2 signaling pathway is a conserved mechanism underlying memory consolidation, reconsolidation, and extinction in key brain areas and in a variety of memory paradigms (Cestari et al., 2014). Its ubiquitous function combined with its activation time course suggest that ERK1/2 is an ideal candidate marker to study the transition from reconsolidation to extinction by increasing the number of nonreinforced CS presentations. Our experiments confirm the requirement for BLA ERK1/2 activation in both the reconsolidation and extinction of fear memory (Duvarci et al., 2005; Herry et al., 2006) and also show for the first time that an intermediate number of CS presentations fails to activate the kinase, leaving it at the level seen in a trained, but not reminded, control group. These data suggest that within a limited range increasing CS exposure terminates the labilization of the original CS-US memory, without necessarily engaging memory extinction. This intriguing finding has several theoretical implications for the neural mechanisms of memory persistence upon retrieval.

In the procedure used here, four CS presentations terminated the incipient memory labilization and restabilization mechanisms triggered by the first CS. We speculate that one or more CSdependent molecular events may mediate this arrest of the earliest reconsolidation intracellular signaling mechanism in an extinction-independent manner. For example, a protein-protein interaction that reduces or buffers $\mathrm{Ca}^{2+}$ flow through the NMDAR could be activated by four CSs to act as an early molecular brake that disengages an ongoing synaptic plasticity mechanisms (Cho et al., 2001). Additionally, given that memory labilization is required for reconsolidation and depends on degradation of preexisting postsynaptic proteins via activation of the ubiquitin proteasome system (Lee et al., 2008), labilization arrest could require the activation of specific deubiquitinating enzymes (DUBs) within the BLA. The DUB ubiquitin C-terminal hydrolase L1 is highly specific to neurons and suppresses TNF- $\alpha$-induced ERK1/2 activation in vitro (Ichikawa et al., 2010).

Animals receiving seven CS presentations also showed lack of BLA ERK1/2 activation, but were nevertheless molecularly and behaviorally sensitive to the effect of NMDAR agonism since DCS potentiated the emerging dominant memory process of extinction. This important difference between two intermediate states highlights both the insensitivity of the intracellular signaling cascade after four CS presentations and also that extinction engagement is a gradual process developing after a sufficient number of CS presentations (between four and seven) and requiring the concerted action of kinases and phosphatases (de la Fuente et al., 2011; Merlo et al., 2014). Systemic DCS administration increased BLA calcineurin levels after seven CS presentations, leading to extinction enhancement, but had no effect on the phosphatase levels after four CSs (Merlo et al., 2014). Even though reconsolidation termination and the gradual engagement of extinction are both CS repetition-dependent events, it remains to be determined whether molecular changes such as ERK1/2 activation occur in the same or different neuronal subpopula- tions within the BLA. We speculate that the CS-dependent early inactivation of ERK1/2 takes place in the fear neuronal ensemble, which stops responding to the CS as a consequence of extinction training. As more unreinforced CSs are presented, extinction neurons may then increase their firing rate in response to the extinguished CS through an ERK1/2-dependent mechanism (Herry et al., 2008). Defining the molecular mechanisms that act to arrest reconsolidation, manifesting as ERK1/2 insensitivity to CS exposure, will be an important next step to test putative interactions between these opposing memory maintenance and inhibition processes.

\section{Clinical implications of memory in limbo}

A widely used and effective treatment for anxiety disorders is exposure therapy. Manipulation of memory content during exposure sessions either by enhancing memory extinction or preventing memory reconsolidation is emerging as a promising development of such treatments (Bowers and Ressler, 2015). In this context, the use of DCS during exposure sessions has shown a positive effect (Guastella et al., 2008; Otto et al., 2010), a negative effect (Smits et al., 2013), or no effect in patients with anxiety disorders (de Kleine et al., 2012). Furthermore, pharmacological treatment in association with traumatic memory reactivation involving CS exposure has also shown positive (Brunet et al., 2008; Kindt and van Emmerik, 2016) or no effect (Wood et al., 2015) in PTSD patients. We suggest that this pattern of results is consistent with the three-component transition between reconsolidation and extinction reported here. Depending on the strength of the maladaptive aversive memory, CS exposure protocols will affect fear memory differently, but invariably the profile will follow the same transitions occurring during the CS exposure space. A pharmacological treatment such as DCS will enhance reconsolidation or extinction when using extreme CS exposure sessions, but will have no effect when CS exposure results in a memory in limbo. Conversely, if cognitive behavioural therapy is combined with an amnestic treatment to disrupt the traumatic memory, unintentional limbo engagement through an extended CS exposure session will leave the target memory in an insensitive state, thereby preventing clinical improvement. The degree of CS exposure resulting in limbo could vary between individuals, perhaps due to differences in learning history, but knowing of the existence of a limbo state both helps to understand contradictory findings and also promote the development of new treatments to enhance exposure therapy.

\section{References}

Alfei JM, Ferrer Monti RI, Molina VA, Bueno AM, Urcelay GP (2015) Prediction error and trace dominance determine the fate of fear memories after post-training manipulations. Learn Mem 22:385-400. CrossRef Medline

Bouton ME (2004) Context and behavioral processes in extinction. Learn Mem 11:485-494. CrossRef Medline

Bowers ME, Ressler KJ (2015) An overview of translationally informed treatments for posttraumatic stress disorder: animal models of pavlovian fear conditioning to human clinical trials. Biol Psychiatry 78:E15-E27. CrossRef Medline

Brunet A, Orr SP, Tremblay J, Robertson K, Nader K, Pitman RK (2008) Effect of post-retrieval propranolol on psychophysiologic responding during subsequent script-driven traumatic imagery in post-traumatic stress disorder. J Psychiatr Res 42:503-506. CrossRef Medline

Cardinal RN, Aitken MRF (2006) ANOVA for the behavioural sciences researcher. Mahwah, NJ: Erlbaum.

Cassini LF, Flavell CR, Amaral OB, Lee JLC (2017) On the transition from reconsolidation to extinction of contextual fear memories. Learn Mem 24:392-399. CrossRef Medline

Cestari V, Rossi-Arnaud C, Saraulli D, Costanzi M (2014) The MAP(K) of 
fear: from memory consolidation to memory extinction. Brain Res Bull 105:8-16. CrossRef Medline

Cho K, Aggleton JP, Brown MW, Bashir ZI (2001) An experimental test of the role of postsynaptic calcium levels in determining synaptic strength using perirhinal cortex of rat. J Physiol 532:459-466. CrossRef Medline

de la Fuente V, Freudenthal R, Romano A (2011) Reconsolidation or extinction: transcription factor switch in the determination of memory course after retrieval. J Neurosci 31:5562-5573. CrossRef Medline

de Kleine RA, Hendriks GJ, Kusters WJ, Broekman TG, van Minnen A (2012) A randomized placebo-controlled trial of D-cycloserine to enhance exposure therapy for posttraumatic stress disorder. Biol Psychiatry 71:962-968. CrossRef Medline

de Kleine RA, Rothbaum BO, van Minnen A (2013) Pharmacological enhancement of exposure-based treatment in PTSD: a qualitative review. Eur J Psychotraumatol. Advance online publication. Retrieved February 21, 2018. CrossRef Medline

Duvarci S, Nader K, LeDoux JE (2005) Activation of extracellular signalregulated kinase- mitogen-activated protein kinase cascade in the amygdala is required for memory reconsolidation of auditory fear conditioning. Eur J Neurosci 21:283-289. CrossRef Medline

Eisenberg M, Kobilo T, Berman DE, Dudai Y (2003) Stability of retrieved memory: inverse correlation with trace dominance. Science 301:11021104. CrossRef Medline

Flavell CR, Lee JL (2013) Reconsolidation and extinction of an appetitive pavlovian memory. Neurobiol Learn Mem 104:25-31. CrossRef Medline

Guastella AJ, Richardson R, Lovibond PF, Rapee RM, Gaston JE, Mitchell P, Dadds MR (2008) A randomized controlled trial of D-cycloserine enhancement of exposure therapy for social anxiety disorder. Biol Psychiatry 63:544-549. CrossRef Medline

Hermans D, Craske MG, Mineka S, Lovibond PF (2006) Extinction in human fear conditioning. Biol Psychiatry 60:361-368. CrossRef Medline

Herry C, Trifilieff P, Micheau J, Lüthi A, Mons N (2006) Extinction of auditory fear conditioning requires MAPK/ERK activation in the basolateral amygdala. Eur J Neurosci 24:261-269. CrossRef Medline

Herry C, Ciocchi S, Senn V, Demmou L, Müller C, Lüthi A (2008) Switching on and off fear by distinct neuronal circuits. Nature 454:600-606. CrossRef Medline

Ichikawa T, Li J, Dong X, Potts JD, Tang DQ, Li DS, Cui T (2010) Ubiquitin carboxyl terminal hydrolase L1 negatively regulates TNFalpha-mediated vascular smooth muscle cell proliferation via suppressing ERK activation. Biochem Biophys Res Commun 391:852-856. CrossRef Medline

Kindt M, van Emmerik A (2016) New avenues for treating emotional memory disorders: towards a reconsolidation intervention for posttraumatic stress disorder. Ther Adv Psychopharmacol 6:283-295. CrossRef Medline

Kindt M, Soeter M, Vervliet B (2009) Beyond extinction: erasing human fear responses and preventing the return of fear. Nat Neurosci 12:256258. CrossRef Medline

Lee H, Kim JJ (1998) Amygdalar NMDA receptors are critical for new fear learning in previously fear-conditioned rats. J Neurosci 18:8444-8454. Medline

Lee JL, Di Ciano P, Thomas KL, Everitt BJ (2005) Disrupting reconsolidation of drug memories reduces cocaine-seeking behavior. Neuron 47: 795-801. CrossRef Medline

Lee JL, Milton AL, Everitt BJ (2006) Reconsolidation and extinction of conditioned fear: inhibition and potentiation. J Neurosci 26:10051-10056. CrossRef Medline

Lee SH, Choi JH, Lee N, Lee HR, Kim JI, Yu NK, Choi SL, Lee SH, Kim H, Kaang BK (2008) Synaptic protein degradation underlies destabilization of retrieved fear memory. Science 319:1253-1256. CrossRef Medline

Mamiya N, Fukushima H, Suzuki A, Matsuyama Z, Homma S, Frankland PW, Kida S (2009) Brain region-specific gene expression activation re- quired for reconsolidation and extinction of contextual fear memory. J Neurosci 29:402-413. CrossRef Medline

Maren S (2015) Out with the old and in with the new: synaptic mechanisms of extinction in the amygdala. Brain Res 1621:231-238. CrossRef Medline

Merlo E, Milton AL, Goozée ZY, Theobald DE, Everitt BJ (2014) Reconsolidation and extinction are dissociable and mutually exclusive processes: behavioral and molecular evidence. J Neurosci 34:2422-2431. CrossRef Medline

Milton AL, Merlo E, Ratano P, Gregory BL, Dumbreck JK, Everitt BJ (2013) Double dissociation of the requirement for GluN2B- and GluN2Acontaining NMDA receptors in the destabilization and restabilization of a reconsolidating memory. J Neurosci 33:1109-1115. CrossRef Medline

Nader K, Schafe GE, Le Doux JE (2000) Fear memories require protein synthesis in the amygdala for reconsolidation after retrieval. Nature 406: 722-726. CrossRef Medline

Otto MW, Tolin DF, Simon NM, Pearlson GD, Basden S, Meunier SA, Hofmann SG, Eisenmenger K, Krystal JH, Pollack MH (2010) Efficacy of $\mathrm{d}$-cycloserine for enhancing response to cognitive-behavior therapy for panic disorder. Biol Psychiatry 67:365-370. CrossRef Medline

Parsons RG, Ressler KJ (2013) Implications of memory modulation for posttraumatic stress and fear disorders. Nat Neurosci 16:146-153. CrossRef Medline

Pavlov IP (1927) Conditioned reflexes; an investigation of the physiological activity of the cerebral cortex. London, UK: Oxford UP.

Paxinos G, Watson C (1998) The rat brain in stereotaxic coordinates, Ed 4. San Diego: Academic.

Pedreira ME, Maldonado H (2003) Protein synthesis subserves reconsolidation or extinction depending on reminder duration. Neuron 38:863-869. CrossRef Medline

Pedreira ME, Pérez-Cuesta LM, Maldonado H (2004) Mismatch between what is expected and what actually occurs triggers memory reconsolidation or extinction. Learn Mem 11:579-585. CrossRef Medline

Pérez-Cuesta LM, Maldonado H (2009) Memory reconsolidation and extinction in the crab: mutual exclusion or coexistence? Learn Mem 16: 714-721. CrossRef Medline

Rouder JN, Speckman PL, Sun D, Morey RD, Iverson G (2009) Bayesian t tests for accepting and rejecting the null hypothesis. Psychon Bull Rev 16:225-237. CrossRef Medline

Schafe GE, Atkins CM, Swank MW, Bauer EP, Sweatt JD, LeDoux JE (2000) Activation of ERK/MAP kinase in the amygdala is required for memory consolidation of pavlovian fear conditioning. J Neurosci 20:8177-8187. Medline

Sevenster D, Beckers T, Kindt M (2014) Prediction error demarcates the transition from retrieval, to reconsolidation, to new learning. Learn Mem 21:580-584. CrossRef Medline

Smits JA, Rosenfield D, Otto MW, Marques L, Davis ML, Meuret AE, Simon NM, Pollack MH, Hofmann SG (2013) D-cycloserine enhancement of exposure therapy for social anxiety disorder depends on the success of exposure sessions. J Psychiatr Res 47:1455-1461. CrossRef Medline

Suzuki A, Josselyn SA, Frankland PW, Masushige S, Silva AJ, Kida S (2004) Memory reconsolidation and extinction have distinct temporal and biochemical signatures. J Neurosci 24:4787-4795. CrossRef Medline

Vervliet B, Craske MG, Hermans D (2013) Fear extinction and relapse: state of the art. Annu Rev Clin Psychol 9:215-248. CrossRef Medline

Wagenmakers EJ (2007) A practical solution to the pervasive problems of $\mathrm{p}$ values. Psychon Bull Rev 14:779-804. CrossRef Medline

Wood NE, Rosasco ML, Suris AM, Spring JD, Marin MF, Lasko NB, Goetz JM, Fischer AM, Orr SP, Pitman RK (2015) Pharmacological blockade of memory reconsolidation in posttraumatic stress disorder: three negative psychophysiological studies. Psychiatry Res 225:31-39. CrossRef Medline 